\title{
dAcsl, the Drosophila Ortholog of Acyl-CoA Synthetase Long-Chain Family Member 3 and 4, Inhibits Synapse Growth by Attenuating Bone Morphogenetic Protein Signaling via Endocytic Recycling
}

\author{
Zhihua Liu, ${ }^{1 \star}$ Yan Huang, ${ }^{\star}{ }^{\star}$ Wen Hu, ${ }^{2}$ Sheng Huang, ${ }^{1}$ Qifu Wang, ${ }^{1}$ Junhai Han, ${ }^{2}$ and Yong Q. Zhang ${ }^{1}$ \\ ${ }^{1}$ Key Laboratory for Molecular and Developmental Biology, Institute of Genetics and Developmental Biology, Chinese Academy of Sciences, Beijing 100101, \\ China, and ${ }^{2}$ Institute of Life Science and the Key Laboratory of Developmental Genes and Human Disease, Southeast University, Nanjing 210096, China
}

\begin{abstract}
Fatty acid metabolism plays an important role in brain development and function. Mutations in acyl-CoA synthetase long-chain family member 4 (ACSL4), which converts long-chain fatty acids to acyl-CoAs, result in nonsyndromic X-linked mental retardation. ACSL4 is highly expressed in the hippocampus, a structure critical for learning and memory. However, the underlying mechanism by which mutations of ACSL4 lead to mental retardation remains poorly understood. We report here that dAcsl, the Drosophila ortholog of ACSL4 and ACSL3, inhibits synaptic growth by attenuating BMP signaling, a major growth-promoting pathway at neuromuscular junction (NMJ) synapses. Specifically, $d A c s l$ mutants exhibited NMJ overgrowth that was suppressed by reducing the doses of the BMP pathway components, accompanied by increased levels of activated BMP receptor Thickveins (Tkv) and phosphorylated mothers against decapentaplegic (Mad), the effector of the BMP signaling at NMJ terminals. In addition, Rab11, a small GTPase involved in endosomal recycling, was mislocalized in $d A c s l$ mutant NMJs, and the membrane association of Rab11 was reduced in $d A c s l$ mutant brains. Consistently, the BMP receptor Tkv accumulated in early endosomes but reduced in recycling endosomes in $d A c s l$ mutant NMJs. dAcsl was also required for the recycling of photoreceptor rhodopsin in the eyes, implying a general role for dAcsl in regulating endocytic recycling of membrane receptors. Importantly, expression of human ACSL4 rescued the endocytic trafficking and NMJ phenotypes of $d A c s l$ mutants. Together, our results reveal a novel mechanism whereby dAcsl facilitates Rab11-dependent receptor recycling and provide insights into the pathogenesis of ACSL4-related mental retardation.
\end{abstract}

Key words: BMP signaling; Drosophila; mental retardation; neuromuscular junction; synapse; vesicle trafficking

\section{Introduction}

Mounting evidence shows that proteins involved in the synthesis and turnover of fatty acids affect brain development and cognitive functions (Najmabadi et al., 2011). Mutations in acyl-CoA synthetase long-chain family member 4 (ACSL4) give rise to non-

\footnotetext{
Received Aug. 19, 2013; revised Jan. 7, 2014; accepted Jan. 8, 2014.

Author contributions: Z.L. and Y.H. designed research; Z.L., Y.H., W.H., S.H., and Q.W. performed research; Z.L., Y.H., W.H., S.H., Q.W., J.H., and Y.Q.Z. analyzed data; Z.L., Y.H., J.H., and Y.Q.Z. wrote the paper.

This work was supported by Ministry of Science and Technology Grant 2014CB942803, Strategic Priority Research Program B of the Chinese Academy of Sciences Grant XDB02020400, and National Science Foundation of China Grant 31110103907 to Y.Q.Z. We thank C. Bökel, G. Davis, P. ten Dijke, H. Krämer, M. González-Gaitán, P. Garrity, X. Lin, A. Nakamura, Z. Wang, T. Xie, and P. Verstreken, the Developmental Studies Hybridoma Bank, and the Bloomington Stock Center for antibodies and flies; Drs. H. Bellen, V. Budnik, M. Crickmore, G. Davis, A. DiAntonio, K. Koles, D. Rogulja, T. Schwarz, Y.Xiong, and particularly A. Rodal for discussions and comments on the manuscript; Dr. J. Liu for advice on subcellular fractionation; and Dr. L. Yang at the Electron Microscopy Facility of the Institute of Genetics and Developmental Biology on EM analysis of NMJ synapses.

The authors declare no competing financial interests.

${ }^{*}$ Z.L. and Y.H. contributed equally to this work.

Correspondence should be addressed to Dr. Yong Q. Zhang, Institute of Genetics and Developmental Biology, Chinese Academy of Sciences, No. 1 Datun Road, Chao Yang District, Beijing 100101, China. E-mail: yqzhang@genetics.ac.cn.

Z. Liu's present address: Department of Neurobiology, Harvard Medical School, Boston, MA 02115.

DOI:10.1523/JNEUROSCI.3547-13.2014

Copyright $\odot 2014$ the authors $\quad 0270-6474 / 14 / 342785-12 \$ 15.00 / 0$
}

syndromic X-linked mental retardation (Meloni et al., 2002). ACSL4 belongs to a family of enzymes that converts long-chain fatty acids to acyl-CoA esters, which serve as important intermediates in lipid biosynthesis and fatty acid degradation (Li et al., 2010), and is enriched in endoplasmic reticulum (ER) (Meloni et al., 2009), the major site for lipid biosynthesis. dAcsl, the Drosophila ortholog of ACSL3 and ACSL4, also localizes to ER (Zhang et al., 2009; O’Sullivan et al., 2012). ACSL4 is highly expressed in the hippocampus, a structure critical for learning and memory (Cao et al., 2000; Meloni et al., 2002), where it is required for dendritic spine formation (Meloni et al., 2009). However, how ACSL4 regulates neural development remains unknown.

Endocytic trafficking of membrane receptors plays an important regulatory role in signal transduction (Sorkin and von Zastrow, 2009). After internalization and delivery to early endosomes, receptors can be sorted either to late endosomes and lysosomes for degradation, or back to the plasma membrane via recycling endosomes for reuse. Distinct trafficking steps are orchestrated by specific GTPases (Stenmark, 2009). For example, Rab11, which preferentially localizes to recycling endosomes, plays an important role in protein recycling from endosomes back to plasma membrane (Grant and Donaldson, 2009). ACSL4 
promotes endocytic degradation of epidermal growth factor receptors (EGFRs) in cultured cells (Zhang et al., 2011). However, little is known about the role of ACSL4 in endosomal trafficking in vivo.

At the Drosophila neuromuscular junctions (NMJ), one of the best characterized signaling pathways is the retrograde BMP cascade that promotes NMJ synapse growth (Collins and DiAntonio, 2007; Fuentes-Medel and Budnik, 2010; Bayat et al., 2011). A number of endosomal proteins, including Spichthyin, endosomal maturation defective (Ema), and Spinster, have been identified as negative regulators of BMP signaling (Sweeney and Davis, 2002; Dermaut et al., 2005; Wang et al., 2007; Kim et al., 2010). Mutations in these genes cause elevated BMP signaling and overgrown NMJ synapses characterized by supernumerary boutons.

The in vivo functions of ACSL4 are well conserved from Drosophila to humans, as the phenotypes of $d A c s l$ mutants can be effectively rescued by expressing human ACSL4 (Zhang et al., 2009; Liu et al., 2011). We previously reported that $d A c s l$ mutants exhibit distally biased axonal transport defects, leading to atrophic NMJs in the posterior muscles innervated by longer axons (Liu et al., 2011). We report here that NMJ terminals in the anterior muscles innervated by shorter axons are however overgrown with upregulated BMP signaling in $d A c s l$ mutants. Furthermore, $d A c s l$ mutations decrease membrane association of Rab11 and alter the synaptic localization of Rab11. Accordingly, endocytic recycling of the BMP receptor Tkv is impaired, and the activated Tkv accumulates in $d A c s l$ mutant NMJs. This study thus establishes a critical role for dAcsl in endosomal trafficking of BMP receptor Tkv during synapse development.

\section{Materials and Methods}

Drosophila strains and genetics. Flies of either sex were cultured in standard cornmeal media at $25^{\circ} \mathrm{C}$ unless otherwise indicated. $w^{1118}$ was used as the wild-type control, unless otherwise indicated. $d A c s l^{8}, d A c s l^{1}, d A c s l^{05847}$, dAcsl ${ }^{K O}$, OK6-Gal4, P\{UAS-Acsl.RNAi\}, P\{UAS-Acsl.715.MycC $\}$, and $P\{U A S-A C S L 4 . L . M y c\}$ were described previously (Zhang et al., 2009; Liu et al., 2011). UAS-dAcsl-RNAi 2 (THU2816) was provided by Tsinghua University RNAi Center (Ni et al., 2009). $g b b^{4}$ and $g b b^{D 20}$ were provided by T. Xie (Stowers Institute for Medical Research). P\{UAS-GFP-myc$2 \times F Y V E\} 2$ (Wucherpfennig et al., 2003) and $P\{U A S-T k v$.GFP (Dudu et al., 2006) were provided by M. González-Gaitán (University of Geneva, Geneva, Switzerland). P\{UAS::TIPF\} (Tkv-InversePericam-FKBP12) was from C. Bökel (Center for Regenerative Therapies Dresden, Dresden, Germany) (Michel et al., 2011). P\{UAS-spin.myc-EGFP $\} B$ and $P\{n-s y b-$ GAL4.S\} were from G. Davis (University of California, San Francisco) (Sweeney and Davis, 2002) and P. Verstreken (VIB Center for the Biology of Disease, Leuven, Belgium) (Verstreken et al., 2009), respectively. The following fly lines were obtained from the Bloomington Stock Center: P\{GAL4-Mhc.W\},P\{GawB\}elav[C155],P\{GAL4\}repo, $P\{t u b P-G A L 4\} L L 7, w i t^{A 12}, w i t^{B 11}, t k v^{7}, \mathrm{dad}^{\mathrm{Jle4}}, \mathrm{rabl1}^{93 B i}, \mathrm{mad}^{\mathrm{Koo237}}$, $P\{U A S p-Y F P . R a b 5\} 02$, and $P\{w[+m C]=$ Gal4-ninaE.GMR $\} 12$.

Immunohistochemical staining. Immunostaining of larval preparations was performed as previously described (Liu et al., 2011). For antibody staining of the proteins Brp, Rab11, and Bchs, larvae were fixed in
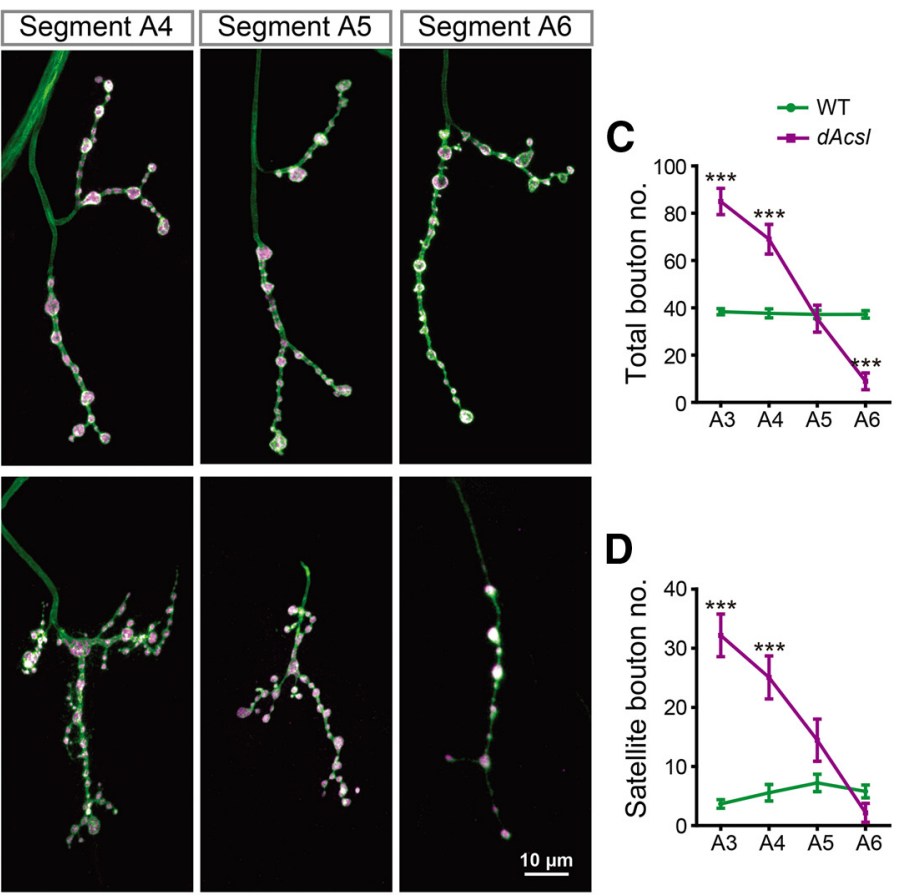

Figure 1. Morphological NMJ phenotypes of $d A c s /$ mutants. $\boldsymbol{A}, \boldsymbol{B}$, Representative confocal images of NMJ4 from abdominal

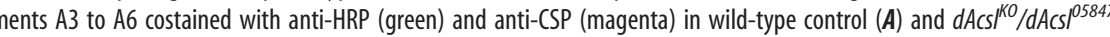
whereas those innervating the posterior segment muscles (i.e., A6) with longer axons were dystrophic. Scale bar, $10 \mu \mathrm{m}$. $\boldsymbol{C}, \boldsymbol{D}$ Quantitative results of the total bouton number $(\boldsymbol{C})$ and satellite bouton number $(\boldsymbol{D}) . n>18$ NMJs for all genotypes. ${ }^{* * *} p<0.001$ (Student's $t$ test). Error bars indicate SEM.

Bouin's solution (Sigma-Aldrich) for $5 \mathrm{~min}$. For other antibodies, specimens were fixed in 4\% paraformaldehyde for 30-60 min. To preserve the YFP fluorescence of the TIPF reporter, PBS without detergent was used for immunostaining. The primary antibodies anti-CSP (6D6, 1:500), anti-Brp (nc82; 1:50), and anti-Myc (9E10; 1:200) were from the Developmental Studies Hybridoma Bank (DSHB) at Iowa. Other antibodies used were as follows: rabbit anti-GFP (1:200; Clontech), rabbit anti-phosphorylated MAD (1:500; P. ten Dijke, Leiden University, Leiden, The Netherlands) (Persson et al., 1998), mouse anti-Rab11 (1:50; BD Biosciences), rat anti-Bchs (1:200; P. Garrity, Brandeis University, Waltham, Massachusetts) (Khodosh et al., 2006), rabbit anti-Rab5 (1:50; M. González-Gaitán, University of Geneva, Geneva, Switzerland) (Wucherpfennig et al., 2003), guinea pig anti-Spinster (1:200; G. Davis, University of California, San Francisco) (Sweeney and Davis, 2002), rabbit anti-Nwk (1:1000; B. Ganetzky, University of Wisconsin-Madison, Madison, Wisconsin) (Coyle et al., 2004), and FITC-conjugated antiHRP (1: 200; Jackson ImmunoResearch Laboratories). The following three antibodies were provided by H. Krämer (University of Texas Southwestern Medical Center, Texas): rabbit anti-Avl/Syx7 (1:1000), guinea pig anti-Dor (1:500) (Pulipparacharuvil et al., 2005), and rabbit anti-Hook (1:1000) (Krämer and Phistry, 1996). All primary antibodies were visualized using specific secondary antibodies conjugated to Fluor488, Cy3 (both at 1:1000; Invitrogen) or DyLight 649 (1:500; Jackson ImmunoResearch Laboratories).

Imaging and data analysis. Images were collected with a Leica TCS SP5 confocal microscope using a $40 \times / 1.25 \mathrm{NA}$ oil objective and LAS AF software or with an Olympus Fluoview FV1000 confocal microscope using a $60 \times / 1.42$ NA oil objective and FV10-ASW software. All images for a specific experiment were captured using identical settings for statistical comparison among different genotypes unless otherwise specified in the figure legends. High-resolution confocal images in Figures 3, 4, and 5 were processed with the deconvolution software AutoQuant X2 (Media Cybernetics). Brightness, contrast, and color were adjusted using Photoshop CS3 (Adobe). Muscle 4 neuromuscular junction (NMJ4) of 
A
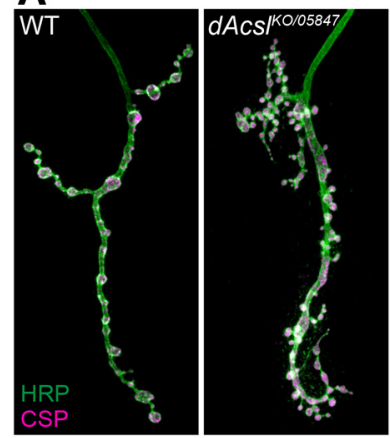

B

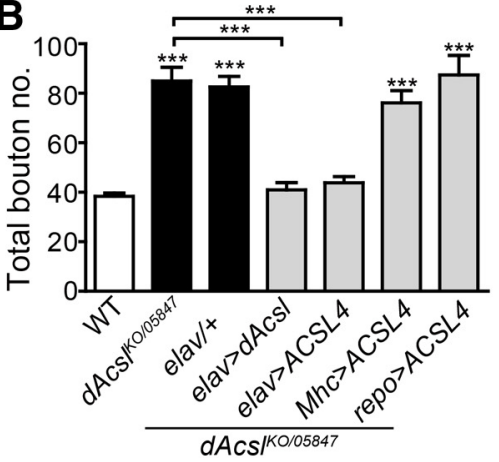

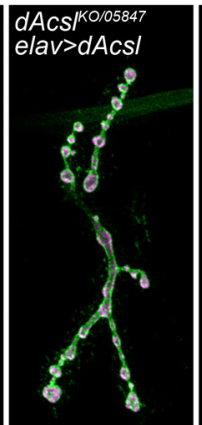
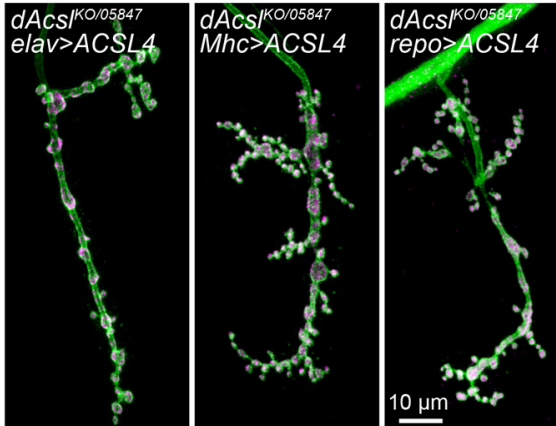

C

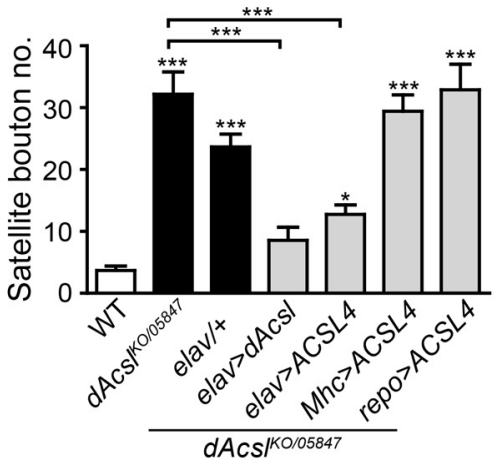

Figure 2. $d A c s /$ inhibits synaptic growth presynaptically. $A$, NMJ4 synapses of abdominal segment $A 3$ were double-labeled with anti-HRP (green) and anti-CSP (magenta) to reveal presynaptic membranes and synaptic vesicles, respectively. Representative NMJ4 synapses of different genotypes are as follows: wild-type, $d A c s /{ }^{K O} / d A c s / 05847$, neuronal rescue by dAcsl and human ACSL4 $\left.(\text { elav-Gal4I+; } d A c s)^{K O} / d A c s\right)^{05847} ;$ UAS-dAcsl/+ and elav-Gal4/+;dAcsl ${ }^{K O} / d A c s{ }^{05847} ;$ UAS-ACSL4I+), muscular rescue (dAcs) $)^{K O}$ dAcs ${ }^{55847}$; Mhc-Gal4/UAS-ACSL4), and glial rescue by human ACSL4 (dAcs/ ${ }^{K O} / d A c s /{ }^{05847}$; repo-Gal4/UAS-ACSL4). Pan-neural expression of dAcsl or human ACSL4 driven by elav-Gal4 rescued the NMJ overgrowth phenotype of $d A$ cs/mutants, but muscular and glial expression of ACSL4 did not. Scale bar, $10 \mu \mathrm{m} . \boldsymbol{B}, \boldsymbol{C}$, Quantitative results of the total bouton number $(\boldsymbol{B})$ and satellite bouton number $\left(\boldsymbol{C}\right.$ ) in different genotypes. $n>18$ for all genotypes. ${ }^{*} p<0.05$ (one-way ANOVA with Tukey post hoc tests). ${ }^{* * *} p<0.001$ (one-way ANOVA with Tukey post hoc tests). Error bars indicate SEM.

abdominal segments A2 and A3 was used for all fluorescence imaging experiments, and all images were analyzed and quantified using ImageJ (National Institutes of Health, Bethesda, Maryland). For quantification of NMJ morphological phenotypes, individual boutons were defined according to the discrete staining signal of anti-CSP. Satellite boutons were defined as extensions of five or fewer small boutons emanating from the main branch of the NMJ terminals (O'Connor-Giles et al., 2008). For quantification of pMad, Rab11, and Tkv levels, mean fluorescence intensities were measured within HRP-positive NMJ4s on abdominal segments A2 and A3. To quantify the colocalization of intracellular TkvGFP-positive puncta and endosomal markers at NMJ4 synapses, the number of double-positive puncta was counted and expressed as a percentage of the total number of Tkv-GFP-positive puncta.

Western analysis and subcellular fractionation. For Western analysis, third instar larval brains and adult heads were homogenized in radioimmunoprecipitation assay buffer (50 mu Tris- $\mathrm{HCl}, 150 \mathrm{~mm} \mathrm{NaCl}, 1 \%$ NP-40, 0.1\% SDS) with proteinase inhibitors (Calbiochem) on ice. Samples were then subjected to SDS-PAGE and immunoblotting according to standard procedures. For subcellular fractionation, third instar larval brains were homogenized in ice-cold homogenization buffer $(10 \mathrm{mM}$ HEPES, $250 \mathrm{~mm}$ sucrose containing protease inhibitor mixture). The nuclei and cell debris were pelleted by centrifugation at $1000 \times g$ for 10 $\mathrm{min}$, and the resulting supernatant was subjected to ultracentrifugation at $100,000 \times g$ for $2.5 \mathrm{~h}$ using a Beckman TLS-55 rotor to generate the cytosol and the pellet, which was resuspended in the equal volume of the cytosolic fraction to represent membrane fraction. Equal volumes of the cytosol and membrane fractions were loaded on an SDS-PAGE gel and analyzed by Western blotting. The following antibodies were used: guinea pig anti-dAcsl (1:3000) (Zhang et al., 2009), mouse anti-syntaxin (8C3; 1:1000, DSHB), mouse anti-Rab11 (1:1000), mouse anti-CSP
(6D6; 1:1000, DSHB), rabbit anti-Rab7 (1: 10,000) (Tanaka and Nakamura, 2008), rabbit anti-Rab5 (1:1000, Abcam), and mouse antiactin (100,000, Millipore Bioscience Research Reagents).

Electron microscopy. Electron microscopy (EM) was performed as described previously (Liu et al., 2011). Briefly, dissected third-instar larvae were fixed overnight at $4^{\circ} \mathrm{C}$ in $1 \%$ glutaraldehyde plus $4 \%$ PFA in $0.1 \mathrm{~m}$ cacodylate buffer, $\mathrm{pH} 7.4$, and postfixed in $1 \% \mathrm{OsO}_{4}$ in cacodylate buffer for $90 \mathrm{~min}$ at room temperature. Samples were then stained in saturated aqueous uranyl acetate for $1 \mathrm{~h}$, dehydrated in a graded acetone series, and embedded in Spurr resin (Electron Microscopy Sciences). Neuromuscular junction 6/7 terminals in abdominal segments A2 and A3 were serially sectioned with a Leica UC6 ultramicrotome, stained with uranyl acetate and Sato's lead, and observed using a JEOL 1400 electron microscope. Large vesicles $>60 \mathrm{~nm}$ in diameter from $>30$ boutons of three wild-type and four $d A c s l^{05847} / d A c s l^{K O}$ larvae were statistically analyzed.

Rh1 recycling analysis. Knockdown of $d A c s l$ in the eye was brought about by GMR-Gal4driven RNAi. Adult eyes containing exclusively mutant cells homozygous for $d A c s l^{K O}$ were generated using the EGUF-hid method (Stowers and Schwarz, 1999). Rh1 endocytosis and recycling were evaluated as previously described (Han et al., 2007; Cao et al., 2011). Briefly, 0- to 2-d-old adult flies were collected and exposed to bright white light (5000 Lux) for $5 \mathrm{~h}$ to induce Rh1 endocytosis. Lightexposed flies were kept in the dark for 0,2 , and $5 \mathrm{~h}$ for Rh1 recycling assays. Fly heads were dissected quickly under dim red light and fixed with $4 \%$ PFA in PBS in complete darkness, dehydrated with a standard acetone dehydration series, and embedded in LR White resin. One-micrometer sections were cut across the top half of the eye. A monoclonal antibody against Rh1 (1:50; DSHB) was used for Rh1 labeling. Rh1 endocytosis and recycling were quantified as the number of endocytic Rh1 particles (ERPs) per rhabdomere at specific time points after exposure to light.

Statistical analysis. All data are presented as mean \pm SEM. Student's $t$ tests were used for statistical comparisons between two groups. Multiple group means were compared by one-way ANOVA with Tukey tests for post hoc pairwise comparisons. All comparisons were between a specific genotype and the control unless otherwise indicated.

\section{Results}

dAcsl regulates NMJ development presynaptically

Our previous work showed that axonal transport is impaired and concomitantly the growth and stability of NMJ synapses in posterior abdominal segments (A6 and A7) are reduced in $d A c s l$ mutants (Liu et al., 2011). However, the NMJ synapses of the anterior abdominal segments $\mathrm{A} 2$ and $\mathrm{A} 3$ in $\mathrm{dAcs} \mathrm{IO}^{\mathrm{KO}} / \mathrm{dAcs} \mathrm{l}^{05847}$ mutants were overgrown characterized by more boutons, particularly more satellite boutons, defined as extensions of five or fewer small boutons emanating from the main branch of NMJ terminals (Fig. 1) (O'Connor-Giles et al., 2008). Wild-type larvae exhibited a relatively constant number of total bouton and satellite boutons from the anterior (A3) to posterior (A6) abdominal segments. However, the number of total boutons and satellite boutons of muscle 4 NMJ (NMJ4) in $d A c s l^{K O}$ / $\mathrm{dAcs} \mathrm{C}^{05847}$ mutants was significantly increased in the anterior $\mathrm{A} 3$ and A4 segments but obviously decreased in the posterior A6 segment 
(Fig. 1). The NMJs in other muscles also showed similar alterations as in muscle 4 . The weaker allelic combination $d A c s l^{8}$ / dAcsl ${ }^{05847}$ had a similar but weaker overgrowth-to-undergrowth NMJ phenotype compared with that in the strong hypomorphic $d A_{c s} l^{K O} / d A c s l^{05847}$ combinations. This study focuses on understanding the mechanisms by which the anterior NMJs in segments A2 and A3 overgrow in dAcsl mutants.

To identify the tissue-specific requirement of dAcsl in NMJ development, we expressed Drosophila dAcsl and human ACSL4 under the control of different Gal4 lines. Neuronal expression of either dAcsl or ACSL4 by elav-Gal4 rescued the increased total and satellite bouton number in NMJ4 of the anterior $d A c s l^{K O} / d A c s l^{05847}$ segments A2 and A3 (Fig. 2), whereas elavGal4 alone, or muscular and glial expression of ACSL4 driven by Mhc-Gal4 and repoGal4, respectively, did not (Fig. 2). These results demonstrate that dAcsl acts specifically in presynaptic neurons to control synaptic growth, and this function is conserved across species from Drosophila to humans. Thus, we used the human ACSL 4 for all subsequent rescue experiments.

\section{dAcsl inhibits synaptic growth by attenuating $\mathrm{BMP}$ signaling}

BMP signaling is a central growthpromoting pathway at developing Drosophila NMJs (Collins and DiAntonio, 2007; Fuentes-Medel and Budnik, 2010; Bayat et al., 2011). The overgrown NMJs in the anterior segments of $d A c s l$ mutants might be caused by upregulated BMP signaling. To test this possibility, we examined genetic interactions between $d A c s l$ and BMP pathway components in regulating synapse growth. Mutations in wishful thinking (wit), which encodes a Type II $\mathrm{BMP}$ receptor, resulted in a significant reduction in bouton number at NMJ4 (Fig. $3 A, B)$. Similar to wit mutants, $d A c s l$ wit double mutants had a significantly reduced bouton number compared with wild-type (Fig. $3 A, B$ ), indicating that NMJ overgrowth in $d A c s l$ mutants is dependent on BMP signaling. Furthermore, heterozygous mutation of wit or $t k v$ (which encodes a type I BMP receptor) had no effect on NMJ growth in wild-type background but significantly suppressed synaptic overgrowth in $\mathrm{dAcs} \mathrm{I}^{\mathrm{KO}} / \mathrm{dAcs} \mathrm{l}^{05847}$ mutants (Fig. $3 A, B$ ). Additionally, a heterozygous mutation in daughters against Dpp (dad), which encodes an inhibitory Smad (Sweeney and Davis, 2002; O'Connor-Giles et al., 2008), significantly increased bouton number in the weaker allelic combination of $d A c s l^{8} / d A c s l^{05847}$, which showed mild synaptic overgrowth (Fig. $3 A, B$ ). We also observed robust dosage-dependent interac-
A
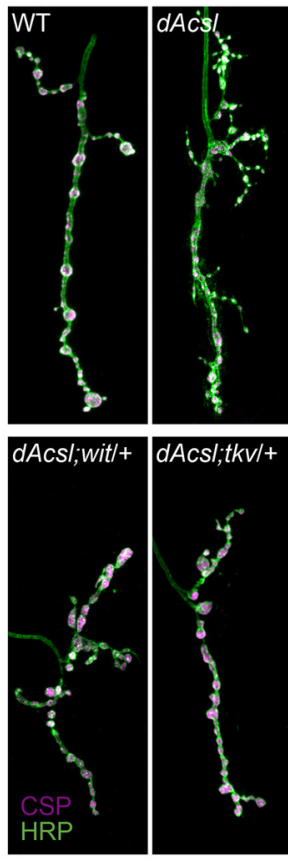

D
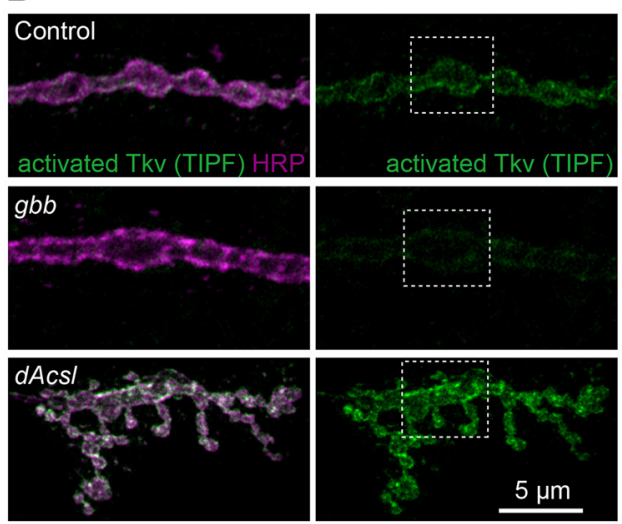

B
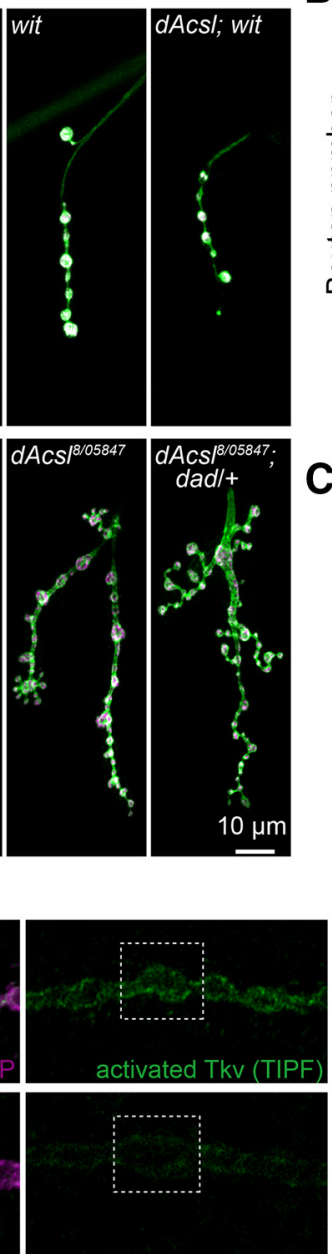

C
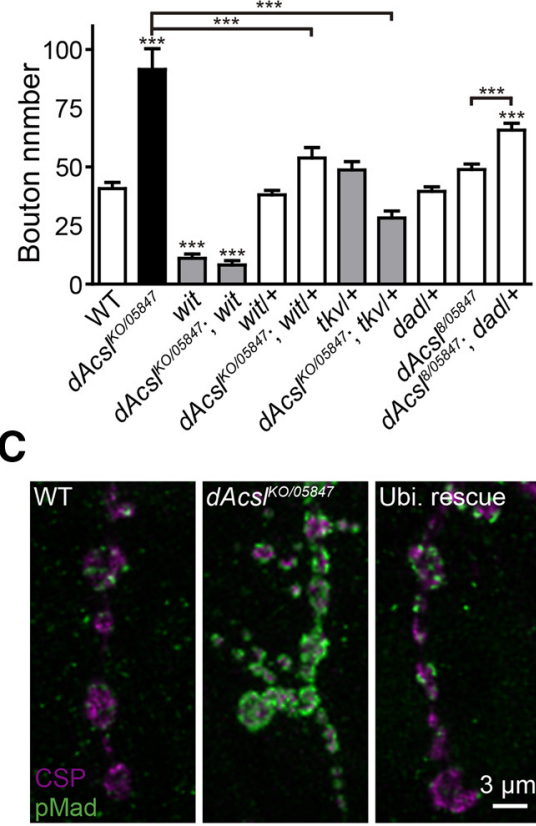

E

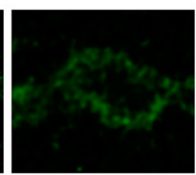

E a-GFP
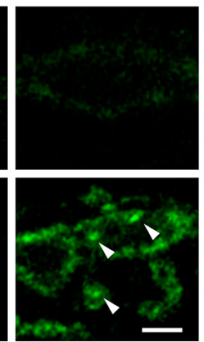

E

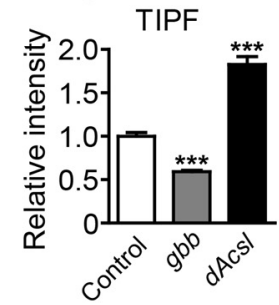

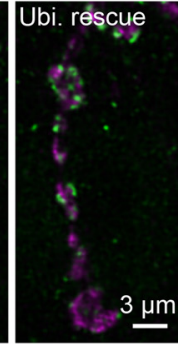

Figure 3. Synaptic overgrowth in $d A c s /$ mutants is caused by elevated BMP signaling. $A$, Confocal images of representative

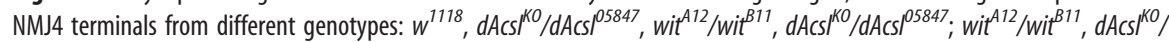

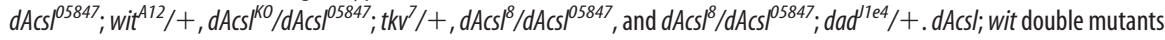
exhibited dystrophic NMJs identical to those observed in wit mutants. Heterozygous wit ${ }^{A 12}$ or $t k v^{7}$ mutations reversed the synaptic overgrowth of $d A c s /$ mutants. The weaker $\left.d A c s\right|^{8} /\left.d A c s\right|^{05847}$ mutants showed a mild increase in bouton number and satellite bouton formation. Heterozygous dad $d^{1 / 24}$ mutation resulted in a significant increase in the synaptic bouton number in $\left.d A c s\right|^{8}$, $d A c{ }^{05847}$ mutants. Scale bar, $10 \mu \mathrm{m}$. $\boldsymbol{B}$, Quantifications of the total bouton number of various genotypes. $n>18$ for all genotypes. ${ }^{* * *} p<0.001$ (one-way ANOVA with Tukey post hoc tests). Error bars indicate SEM. C, NMJ4 synaptic boutons were

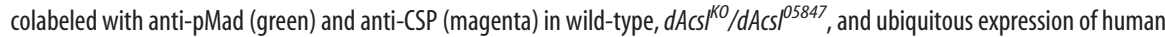
ACSL4 in $d A c s /$ mutant background (dAcs/ $/$ /dAcs ${ }^{05847}$; tub-Gal4/UAS-ACSL4). pMad intensity was markedly increased in $d A c s /$ mutant NMJs, and the increased pMad intensity was completely rescued by ubiquitous expression of human ACSL4. Scale bar, 3 $\mu \mathrm{m}$. D, A fluorescence-based reporter TIPF for activated Tkv receptors was neuronally expressed by $n S y b$-Gal4 in control, $g b b^{4} /$ $g b b^{D 20}$, and $\left.d A c s\right|^{K O} / d A c s{ }^{05847}$ mutants. To preserve the YFP fluorescence of the TIPF reporter, samples were processed without detergent. Right column images, Single confocal slices of that shown in the middle column. Arrowheads indicate TIPF puncta. Scale bars: middle, $5 \mu \mathrm{m}$; right, $1 \mu \mathrm{m}$. $\boldsymbol{E}$, Quantification of the level of total Tkv receptors by anti-GFP staining in different genotypes. $\boldsymbol{F}$, Quantification of the normalized intensity of TIPF against anti-HRP staining in different genotypes. $n>12$ for each genotype. ${ }^{* * *} p<0.001$ (one-way ANOVA with Tukey post hoc tests). Error bars indicate SEM.

tions between $d A c s l$ and other components of the BMP signaling pathway, such as $g b b$ and mad (data not shown). These data provide compelling genetic evidence that synaptic overgrowth in dAcsl mutants depends on the level of BMP signaling.

The Smad family transcription factor Mothers against decapentaplegic (Mad) is an effector of BMP signaling in Drosophila 
A
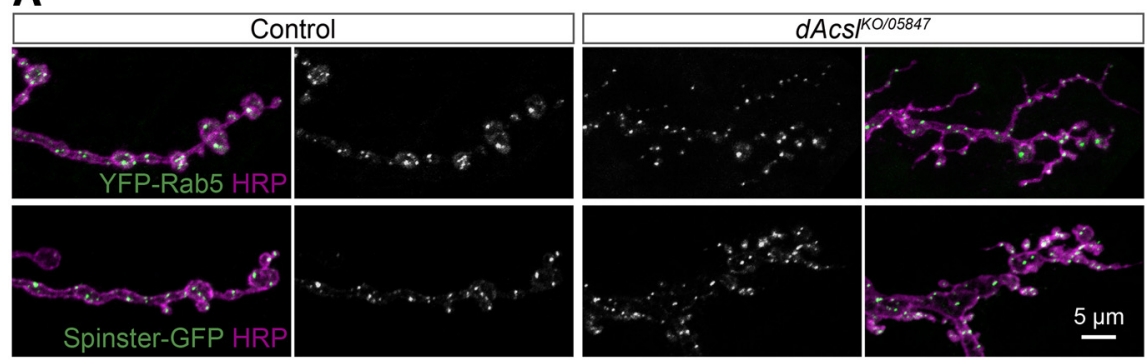

B
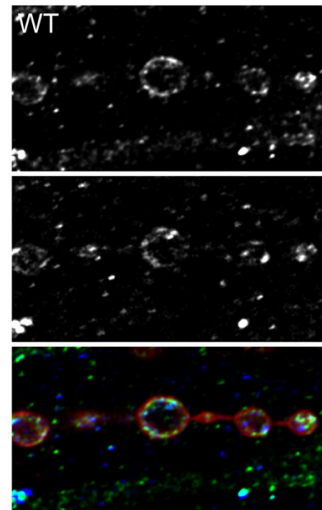

C

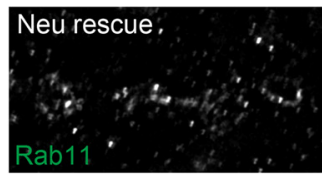

$\mathbf{F}$

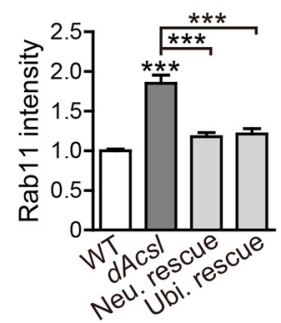

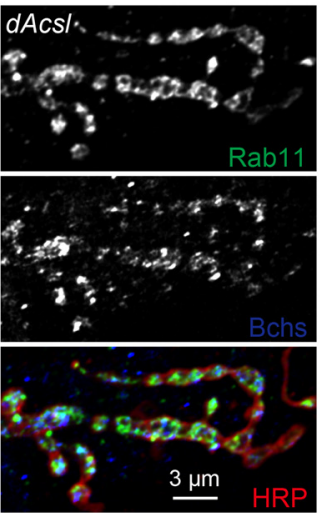

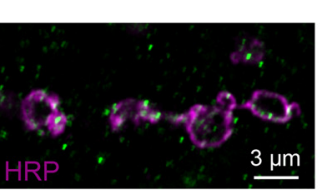

G

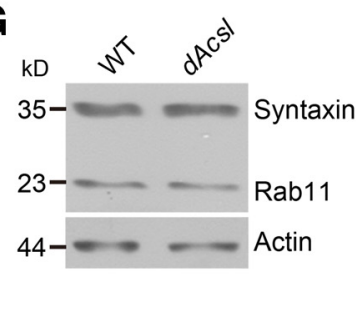

E
D
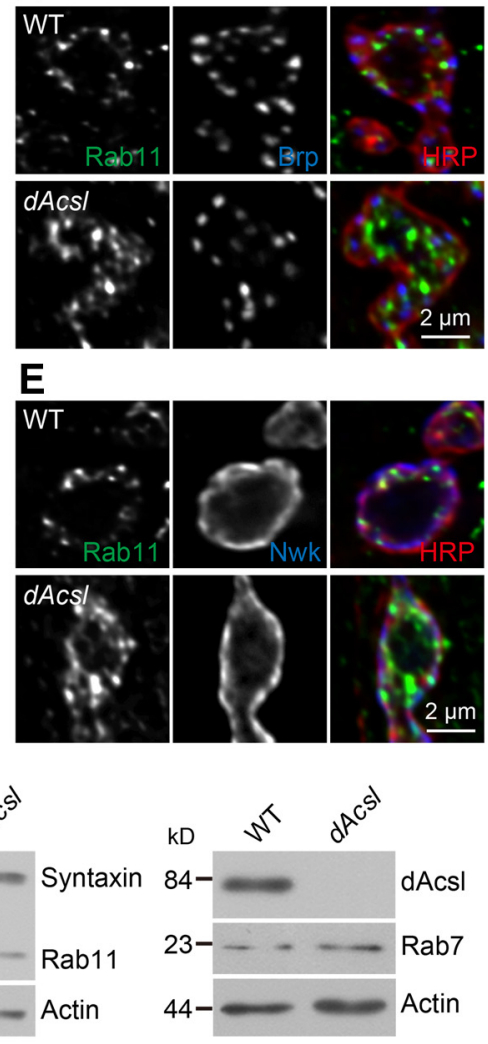

Figure 4. Localization of Rab11 is altered at $d A c s / N M J$ synapses. $A$, Representative confocal images of NMJ4 from control (left) and $\left.d A c s\right|^{K O} /\left.d A c s\right|^{05847}$ mutants (right). YFP-Rab5 (early endosomal) and Spinster-GFP (late endosomal/lysosomal) were expressed presynaptically by elav-Gal4 (green). Neuronal membranes were labeled by anti-HRP (magenta). The staining patterns of these endosomal markers were largely normal at $d A c s$ / mutant NMJs. Scale bar, $5 \mu \mathrm{m} . \boldsymbol{B}, \boldsymbol{C}$, Images represent single confocal slices of NMJ4 colabeled with anti-HRP (red), and recycling endosome markers anti-Rab11 (green) and anti-Bchs (blue) in wild-type, $\left.d A c s\right|^{K O} /\left.d A c s\right|^{05847}$ mutants $(\boldsymbol{B})$, and neuronally rescued larvae $\left(\boldsymbol{C} ;\left.d A c s\right|^{K O} /\left.d A c s\right|^{05847}\right.$; elav-Gal4/UAS-ACSL4). The staining pattern of Rab11 and Bchs was altered in $d A c s /$ mutant boutons $(\boldsymbol{B})$, and the alteration was fully rescued by presynaptic expression of human ACSL4 (C). Scale bar, $3 \mu \mathrm{m}$. D, E, Confocal images showing the localization of Rab11 at synaptic boutons of wild-type and $d A c s K^{K O} /\left.d A c s\right|^{05847}$ as related to $\operatorname{Brp}(\boldsymbol{D})$ and Nwk $(\boldsymbol{E})$. Scale bar, $2 \mu \mathrm{m}$. $\boldsymbol{F}$, Quantification of normalized intensity of Rab11 within the anti-HRP-positive area. $n>18$ NMJs for each genotype. ${ }^{* * *} p<0.001$ (one-way ANOVA with Tukey post hoc tests). Error bars indicate SEM. G, Western results of larval brain extracts of wild-type and $d A c s /{ }^{K O} / d A c s /{ }^{05847}$ detected with antibodies against Rab11, dAcsl, and Rab7. Syntaxin and actin were used as loading controls.

motoneurons, and the level of phosphorylated Mad (pMad) at NMJ terminals has been used as an indicator of BMP signaling (O'Connor-Giles et al., 2008; Shi et al., 2013). pMad level was significantly increased in the anterior NMJs of $d A c s l$ mutants (the relative pMad intensity was $1.00 \pm 0.06$ for wild-type and $4.26 \pm$ 0.45 for $d A c s l^{K O / 05847}$ mutants; $p<0.001$ ), and this increase was suppressed by $t u b$-Gal4-mediated ubiquitous expression of human ACSL4 (1.98 \pm 0.17 for rescued mutants; $p<0.001$ compared with dAcsl mutants; Fig. 3C). pMad staining was also elevated in $d A c s l$ mutant NMJs of the posterior segment A6, although in some small posterior NMJs, pMad staining was absent (data not shown), possibly because these NMJs underwent degeneration (Liu et al., 2011). In light of these dosagesensitive interactions between $d A c s l$ and the BMP signaling pathway components and the increased level of pMad in $d A c s l$ mutants, we conclude that the overgrown NMJ synapses in $d A c s l$ mutants are caused by upregulated BMP signaling.

The level of activated BMP receptor Tkv is elevated in $\boldsymbol{d A c s l}$ mutants

At the Drosophila NMJ, a number of mutants with endocytic trafficking defects show elevated BMP signaling and synaptic overgrowth (Sweeney and Davis, 2002; Wang et al., 2007; O'Connor-Giles et al., 2008; Kim et al., 2010; Rodal et al., 2011; Nahm et al., 2013). We hypothesized that the elevated BMP signaling in $d A c s l$ mutants might be caused by trafficking defects of BMP receptors. If so, there might be an accumulation of activated BMP receptors. We used the fluorescencebased reporter Tkv-InversePericam-FKBP12 (TIPF), in which Tkv is fused to a conformation-sensitive YFP (Michel et al., 2011) to directly monitor the level of phosphorylated, activated Tkv receptors in NMJ synapses. The fluorescence of the conformation-sensitive YFP in TIPF is suppressed when Tkv receptors are inactive, but BMP binding and subsequent Tkv phosphorylation allow YFP to adopt a fluorescent conformation (Michel et al., 2011).

The TIPF reporter was expressed in motoneurons under the control of the stronger $n S y b$-Gal4, which allows better visualization of the YFP signals than the elav-Gal4 and OK6-Gal4-driven TIPF. In wild-type animals, YFP fluorescence overlapping with anti-HRP signals was detected at synaptic boutons (Fig. 3D). YFP intensity at NMJ synapses was reduced in hypomorphs of $g b b$, which encodes the $\mathrm{BMP}$ receptor ligand glass bottom boat (Gbb), validating the usefulness of this reporter in monitoring the level of activated Tkv receptors (Fig. $3 D, F$ ). In $d A c s l^{K O} /$ $d A c s l^{55847}$ mutants, however, the level of activated Tkv, as measured by YFP fluorescence, was significantly increased at NMJs in the anterior segments A2 and A3 (Fig. $3 D, F)$. Interestingly, there were bright YFP puncta internal to the bouton (Fig. 3D, arrowheads), as might be expected for an endosomal structure where the BMP receptors can maintain and even maximize their signaling activity (Wang et al., 2007). As a control, we did not observe obvious changes in the protein levels of the TIPF reporters in either $g b b$ or $d A c s l$ mutant NMJs detected by immunostaining with anti-GFP antibodies, which recognize 
A
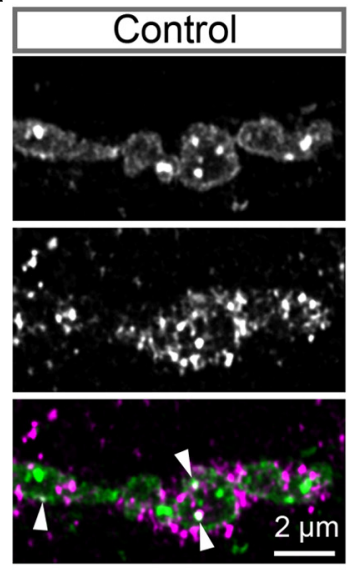

C
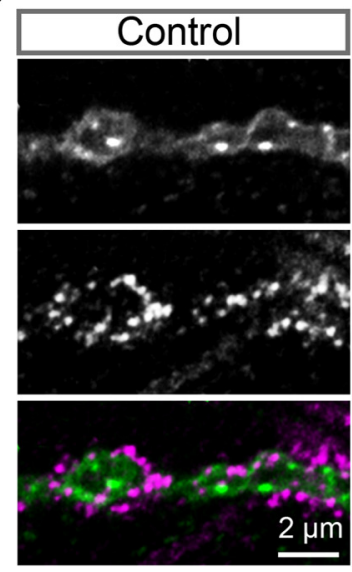

E

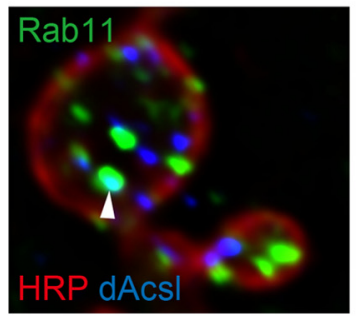

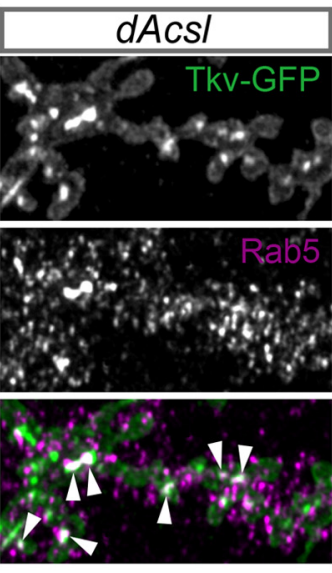
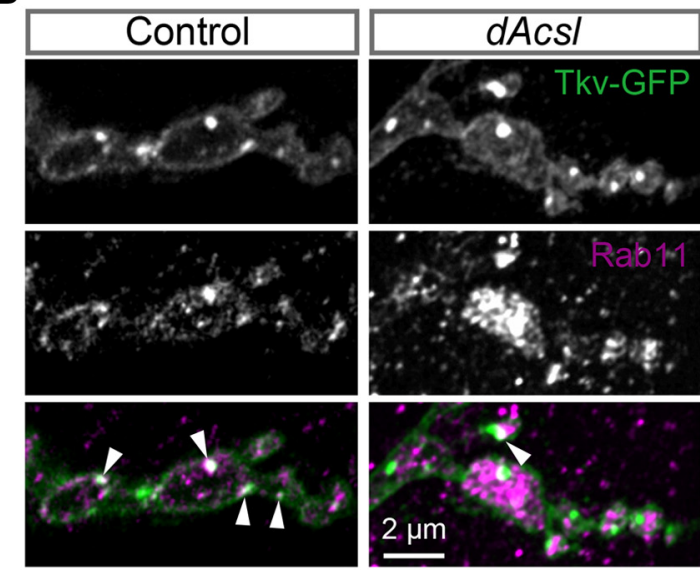

D
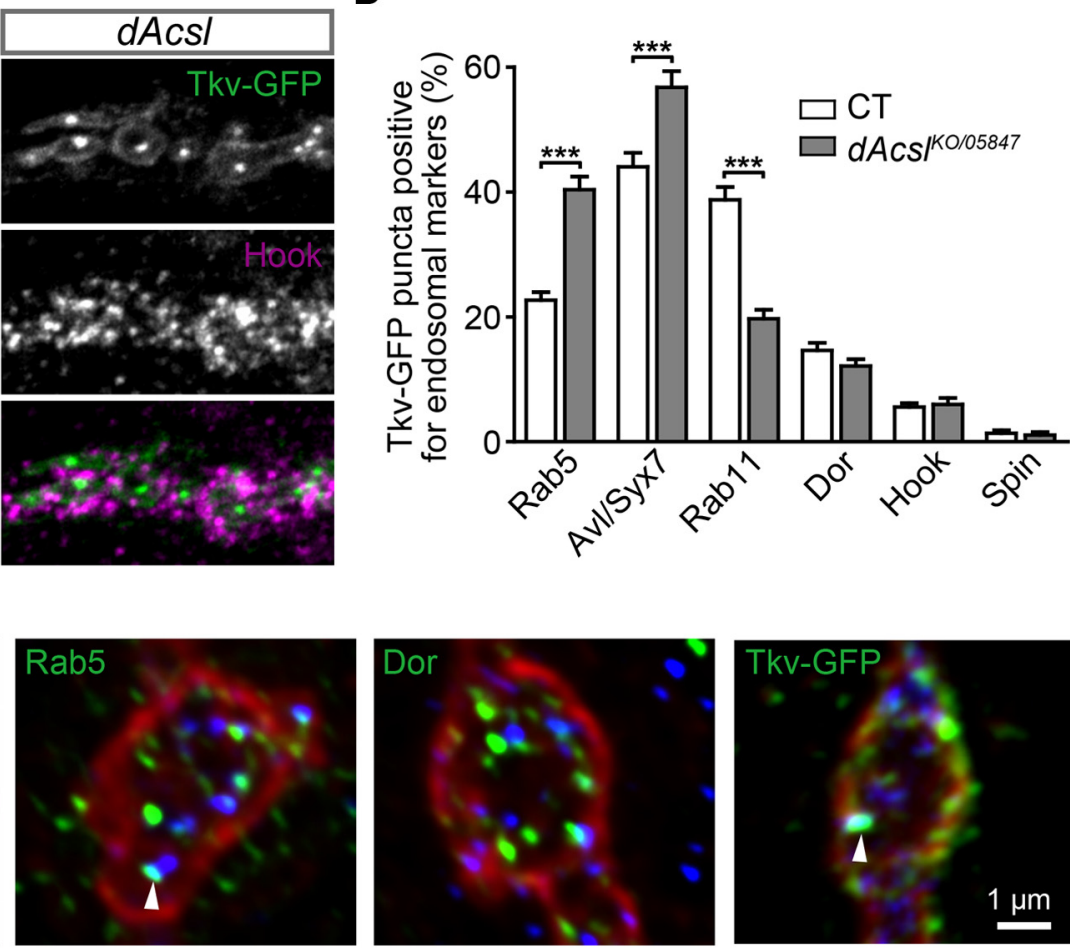

Figure 5. dAcsl affects Tkv trafficking from early endosomes to recycling endosomes. $\boldsymbol{A}-\boldsymbol{C}$, Single-slice confocal images showing colocalization between Tkv-GFP puncta (green) and endosomal markers (magenta): anti-Rab5 (A), anti-Rab11 (B), and anti-Hook (C) in genetically matched control (nSyb-Gal4/UAS-Tkv-GFP) and dAcs/ mutants (dAcs/ ${ }^{K 0}$ UAS-Tkv-GFP/dAcs/ ${ }^{05847}$ nSyb-Gal4). All images were processed by deconvolution. GFP was localized to the presynaptic membrane and discrete intrabouton puncta in both control and mutant boutons. In $d A c s /$ mutants, the percentage of Tkv-GFP-positive puncta costained with early endosomal Rab5 was increased $(\boldsymbol{A})$, but that with the recycling endosomal Rab11 was reduced (B). Arrowheads indicate examples of colocalization of GFP spots with endosomal markers. Few Tkv-GFP puncta colocalized with the late endosomal Hook in both control and mutant synapses (C). Scale bar, $2 \mu \mathrm{m}$. $\boldsymbol{D}$, Quantification of the percentages of Tkv-GFP puncta positive for various endosomal markers. $n>12$ animals for each genotype. ${ }^{* * *} p<0.001$ (Student's $t$ test). Error bars indicate SEM. $\boldsymbol{E}$, Single-slice confocal images showing double-staining of dAcsl-Myc (blue) with Rab11, Rab5, Dor, and Tkv-GFP (green) in NMJ boutons, defined by anti-HRP (red) staining. Motoneuron specific expression of dAcsl-Myc and Tkv-GFP was driven by OK6-Gal4. Arrowheads indicate colocalization of dAcsl with Rab11, Rab5, and Tkv-GFP. Scale bar, $1 \mu \mathrm{m}$.

the GFP variant YFP (Fig. 3E), indicating that the increased level of activated $\mathrm{Tkv}$ receptors in the mutants was not caused by changes in the protein level of the reporter within NMJs. The elevated TIPF signals were also observed in NMJs on the posterior segment A6 (the relative TIPF intensity was $1.00 \pm 0.21$ for control and $1.60 \pm 0.33$ for $d A c s l^{K O /} d A c s l^{05847}$ mutants; $p<0.001$ ), supporting that $\mathrm{dAcsl}$ inactivates $\mathrm{BMP}$ receptors at NMJ terminals across different segments.

\section{dAcsl regulates the distribution of Rab11 at NMJs}

To unravel a possible endocytic trafficking defect in $d A c s l \mathrm{mu}-$ tants, we examined various endosomal markers at NMJ termi- nals. We first examined the distribution of YFP-Rab5 and 2xFYVE-GFP, which label early endosomes (Wucherpfennig et al., 2003), and Spinster-GFP, which labels late endosomes/lysosomes (Sweeney and Davis, 2002; Dermaut et al., 2005). The staining pattern of these markers in the anterior NMJ4 synapses of abdominal segments A2 and A3 was similar between dAcsl mutants and controls (Fig. 4A). Immunostaining with antibodies against Rab5 and the late endosome markers Dor, Hook, and Spinster also showed normal pattern at NMJ terminals of $d A c s l$ mutants (see below).

In contrast, the staining pattern of Rab11 is markedly altered in $d A c s l$ mutant NMJs. In wild-type, Rab11 was located predom- 
A
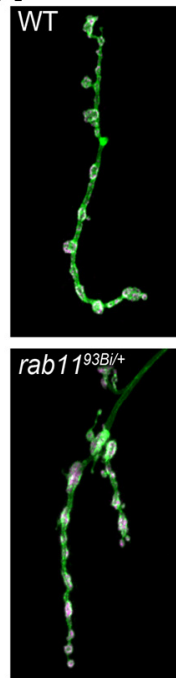

B

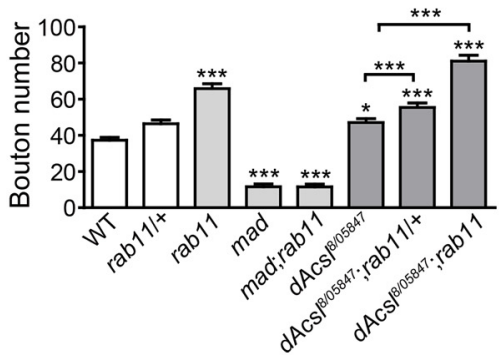

C
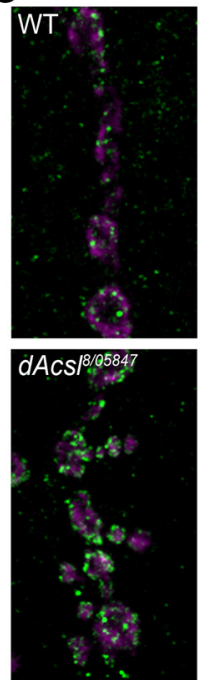

D

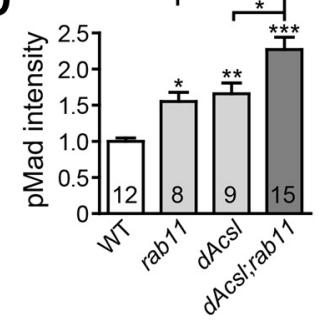

Figure 6. $d A c s /$ synergistically interacts with rab11 to inhibit BMP signaling. $\boldsymbol{A}$, Representative confocal images of NMJ4 stained with anti-HRP (green) and anti-CSP (magenta) in wild-type, $\operatorname{rab}^{193 B i}, \operatorname{mad}^{K 00237}, \operatorname{mad}^{K 00237} ; \operatorname{rab}^{193 B i}, \operatorname{rab}^{933 B i} /+,\left.d A c s\right|^{8} /$

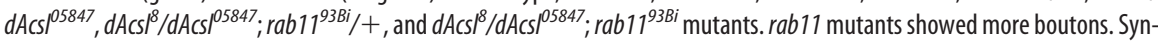
aptic undergrowth in rab11; mad double mutants were indistinguishable from that of mad single mutants (top). Synaptic overgrowth in $\left.d A c s\right|^{8} /\left.d A c s\right|^{05847}$ mutants was enhanced by rab11 mutations (bottom). $\boldsymbol{B}$, Quantification of the total bouton number in the various genotypes. C, NMJ4 synaptic boutons double-labeled with anti-pMad (green) and anti-CSP (magenta) in wild-type, $r a b 11^{93 B i},\left.d A c s\right|^{8} /\left.d A c s\right|^{05847}$, and $\left.d A c s\right|^{8} /\left.d A c s\right|^{05847} ; r^{2} 1611^{93 B i}$ double mutants. Scale bar, $3 \mu \mathrm{m}$. D, Quantification of normalized pMad intensities at NMJ synapses of different genotypes. The number of larvae analyzed is indicated in the columns. ${ }^{*} p<0.05$ (one-way ANOVA with Tukey post hoc tests). ${ }^{* *} p<0.01$ (one-way ANOVA with Tukey post hoc tests). ${ }^{* *} p<0.001$ (one-way ANOVA with Tukey post hoc tests). Error bars indicate SEM.

inantly near the presynaptic membrane (Fig. $4 B, D, E)$, consistent with a previous report (Koles et al., 2012). In $d A c s l^{K O} / d A c s l^{05847}$ mutant boutons, however, Rab11-positive puncta were enlarged and displaced from the presynaptic membrane into the bouton lumen (Fig. 4B,D,E). Similarly, Blue cheese (Bchs), an Rab11 antagonist that extensively overlaps with Rab11 at Drosophila NMJs (Khodosh et al., 2006), also accumulated in $d A c s l^{K O}$ / $d A c s l^{05847}$ mutant boutons (Fig. $4 B$ ). The extended presynaptic distribution of Rab11 was completely reversed by both presynaptic and ubiquitous expression of human ACSL4 (Fig. 4C,F), confirming that the abnormal Rab11 localization is specifically caused by $d A c s l$ mutations. Rab11 did not colocalize with the active zone component Bruchpilot (Brp) (Fig. 4D) and was found adjacent but not overlapping with Nervous wreck (Nwk) (Fig. $4 E$ ), a periactive zone protein. The accumulation of Rab11, observed at $d A c s l$ NMJ terminals of different muscles in different segments, was probably not caused by an elevated expression of Rab11 protein, as Western analysis of larval brains showed a normal level of Rab11, as well as Rab7 protein (Fig. 4G). The altered localization of Rab11 but not other endocytic markers indicates that the function of recycling endosomes may be specifically impaired at $d A c s l$ mutant NMJs.
dAcsl regulates endocytic trafficking of the $\mathrm{BMP}$ receptor $\mathrm{Tkv}$

To directly examine whether the trafficking of BMP receptors at $d A c s l$ mutant NMJs was affected, we expressed a fully functional Tkv-GFP fusion protein (Dudu et al., 2006) in motor neurons. Tkv-GFP was present both at the presynaptic membrane and in puncta throughout synaptic boutons in both controls and $d A c s l^{K O}$ / dAcs ${ }^{05847}$ mutants (Fig. 5A-C), similar to previous observations (Dudu et al., 2006; Kim et al., 2010; Rodal et al., 2011). The synaptic localization of Tkv-GFP-positive puncta was analyzed by double staining with various stage-specific endosomal markers. In both controls and $d A c s l$ mutants, few Tkv-GFP puncta at $d A c s l^{K O}$, $d A c s l^{05847}$ NMJ4 of abdominal segments $\mathrm{A} 2$ and $\mathrm{A} 3$ were positive for the late endosomal/lysosomal markers Dor, Hook, or Spin (Fig. 5C,D). However, dAcsl mutations increased colocalization between Tkv-GFP and the early endosomal markers Rab5 and Avl/Syx7 (Fig. 5A,D) but significantly decreased colocalization with Rab11-positive recycling endosomes, even though the distribution of Rab11 was expanded at the mutant NMJs (Fig. $5 B, D$ ). These data indicate that the trafficking of $\mathrm{BMP}$ receptors from early endosomes to recycling endosomes was specifically impaired in $d A c s l$ mutants. The impaired trafficking of Tkv receptors from early endosomes to recycling endosomes is consistent with the observation that the distribution of Rab11 is specifically altered at $d A c s l$ mutant NMJs (Fig. 4).

In addition, neuronal expression of dAcsl-Myc under the control of OK6Gal4, which can rescue the abnormal NMJ morphology and distribution of Rab11 in dAcsl mutants (data not shown), partially overlapped with Rab11, Rab5, and TkvGFP (Fig. 5E) in the NMJ boutons, suggesting that dAcsl might locally regulate the endocytic trafficking of Tkv receptors.

\section{$d A c s l$ genetically interacts with rab11 in attenuating BMP signaling at NMJ synapses}

The aforementioned data support a model that dAcsl attenuates BMP signaling through facilitating Rab11-dependent BMP receptor recycling. However, whether Rab11 regulates BMP signaling at Drosophila NMJs has not been previously examined. rab11 mutants exhibit synaptic overgrowth with excess satellite boutons (Khodosh et al., 2006), which we confirmed (Fig. 6A,B). Neuronal overexpression of a dominant-negative form of Rab11 driven by elav-Gal4 recapitulated the overgrown NMJs of rab11 mutants (data not shown), suggesting that Rab11 is required in presynaptic neurons to inhibit synaptic growth. Although the synaptic overgrowth in rab11 mutants could not be suppressed by reducing the dose of mad or $t k v$ by half (data not shown), rab11 mad double mutants showed undergrown NMJ synapses that were indistinguishable from those of homozygous mad mutants (Fig. 6A,B), suggesting that mad is downstream of rab11. In 
agreement with the overgrown NMJs, pMad levels were increased at rab11 synapses (Fig. 6C,D). Hence, similar to dAcsl, Rab11 inhibits NMJ growth, probably by attenuating BMP signaling via endocytic recycling.

We next examined genetic interactions between $d A c s l$ and rab11. As strong hypomorphic combinations of $d A c s l^{k o} / d A c s l^{05847}$ $\left(d A c s l^{k o}\right.$ is a null allele) (Liu et al., 2011) in conjunction with a heterozygous, hypomorphic rab1 $1^{93 B i}$ did not survive to wandering third instar larvae, we used weaker allelic combinations of $d A c s l^{8} / d A c s l^{05847}$ (both alleles are hypomorphic) for genetic assays. Overgrowth of NMJs in $d A c s l^{8}$ / $d A c s l^{05847}$ mutants was significantly increased by heterozygosity for the hypomorphic rab $11^{93 B i}$ and further increased when this mutation was homozygous, whereas heterozygosity of $r a b 11^{93 B i}$ alone did not alter NMJ growth (Fig. 6A, B). Furthermore, homozygous rab11 $1^{93 B i}$ mutation significantly increased pMad levels in $\mathrm{AAcsl}^{8} / \mathrm{dAcs} \mathrm{C}^{05847}$ NMJ synapses (Fig. 6C,D). These dosagedependent interactions between $d A c s l$ and rab11 in inhibiting synapse growth and BMP signaling are consistent with the notion that synaptic overgrowth in $d A c s l$ mutants results from compromised Rab11-dependent endosomal recycling.

\section{dAcsl regulates membrane association of Rab11}

The altered staining pattern of Rab11 in $d A c s l$ mutants (Figs. 4 and 5) might be caused by abnormal recycling endosomes labeled by Rab11, mislocalized Rab11 per se, or both. To differentiate these possibilities, we examined NMJ terminals by electron microscopy. Although various membrane organelles accumulate in $d$ Acsl mutant axons in a distally biased fashion because of transport defects (Liu et al., 2011), we observed a normal profile of membrane organelles in $d A c s l$ mutant NMJ boutons of the abdominal segments A2 and A3 (Fig. 7A) where axonal jams are not apparent. Specifically, the number of large vesicles $>60 \mathrm{~nm}$ in diameter, which accumulate in mutants with defects in Rab11dependent vesicle trafficking ( $\mathrm{Li}$ et al., 2007; Otani et al., 2011), was normal in $d A c s l$ mutant boutons $(0.75 \pm 0.09$ large vesicles per $\mu \mathrm{m}^{2}$ cross section of wild-type bouton and $1.23 \pm 0.26$ large vesicles for $d A c s l$ mutants; $p>0.05$ ). These results indicate that Rab11 per se, but not recycling endosomes, may be mislocalized in $d$ Acsl mutant NMJs.

Rab proteins switch between an inactive GDP-bound and an active GTP-bound form, and these changes in activity are coupled to reversible association with target membranes. The membrane association of Rab proteins is critical for their proper functioning (Seabra and Wasmeier, 2004). To understand the biochemical basis for the altered localization of Rab11 in $d A c s l$ mutants, we analyzed the distribution of Rab1 1 in membrane and cytosol fractions of larval brain lysates by subcellular fractionation followed by Western analysis. As a control, the early endosome protein Rab5 and the synaptic vesicle-associated protein CSP were located mostly in the membrane fraction of both wild-type and $d A c s l$ mutant lysates (Fig. $7 B$ ). However, the distribution of Rab11 in membrane and cytosol fractions was signif- icantly altered in $d A c s l$ mutant larval brains (Fig. $7 B$ ); $60 \%$ of Rab11 was associated with membrane in $d A c s l$ mutant brains compared with $80 \%$ in wild-type brains (Fig. $7 C$ ). The decreased membrane association of Rab11 in dAcsl mutant brains was completely rescued by ubiquitous expression of ACSL4 in $d A c s l$ mutants (Fig. $7 B, C$ ). These data indicate that the membrane association of different Rabs is differentially regulated by dAcsl. The reduced membrane association of Rab11, consistent with the expanded distribution pattern of Rab11 in $d A c s l$ mutant NMJs (Fig. $4 B$ ), might impair the endocytic recycling of BMP receptors.

\section{dAcsl promotes rhodopsin recycling in photoreceptors}

To further examine whether dAcsl regulates receptor recycling specifically at NMJ terminals and/or generally in other systems, we used the well-established Drosophila phototransduction model system. Drosophila phototransduction is mediated by a prototypic GTP-binding protein-coupled receptor cascade that is initiated by light activation of rhodopsin. Photoreceptor rhodopsin Rh1 undergoes activity-dependent endocytosis to drive desensitization (Satoh and Ready, 2005; Han et al., 2007), and the endocyotosed rhodopsin is either recycled back to the rhabdomere membrane for resensitization or transported to lysosomes for degradation (Han et al., 2007; Cao et al., 2011). Knocking down of $d A c s l$ in the eyes significantly reduced the recycling rate of ERPs induced by light stimulation (Fig. $8 A-C$ ). Similar results were observed when another independent RNAi line was used and in $\mathrm{AAcs}^{\mathrm{KO}}$ mosaic eyes (Fig. $8 \mathrm{~B}, \mathrm{C}$ ), supporting the notion that $\mathrm{dAcsl}$ is required for $\mathrm{Rh} 1$ recycling. Western analysis revealed that Rh1 protein levels were normal during the light stimulation and recovery process in each genotype (Fig. $8 D$ ), suggesting that most internalized Rh1 was recycled back to the rhabdomere. The recycling process was Rab11-dependent, as only $29.12 \%$ of ERPs disappeared after $2 \mathrm{~h}$ recovery in the adult eye expressing a 
A
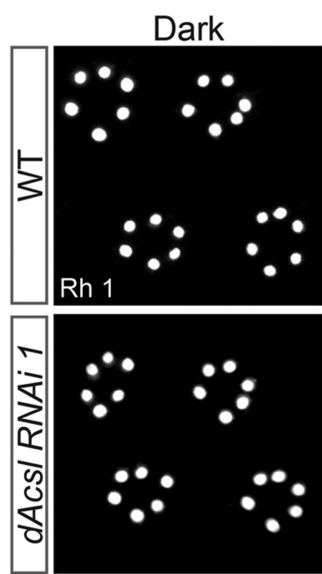

B

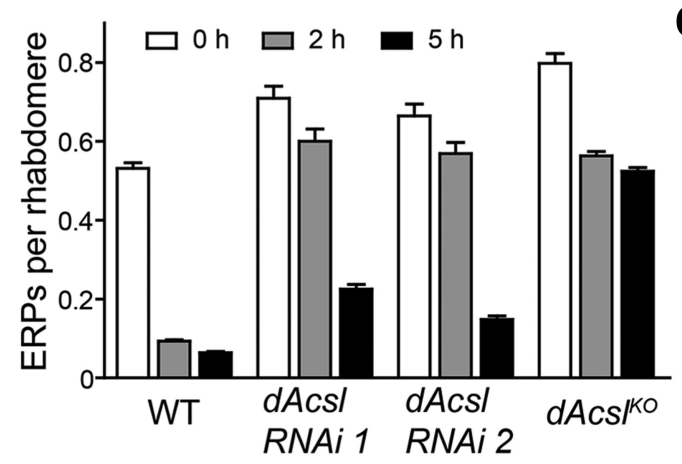

D

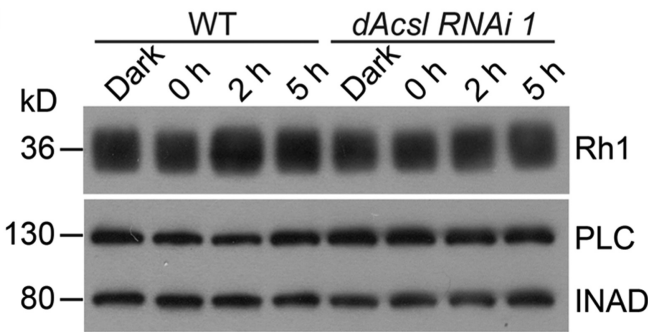

Recovery after light exposure
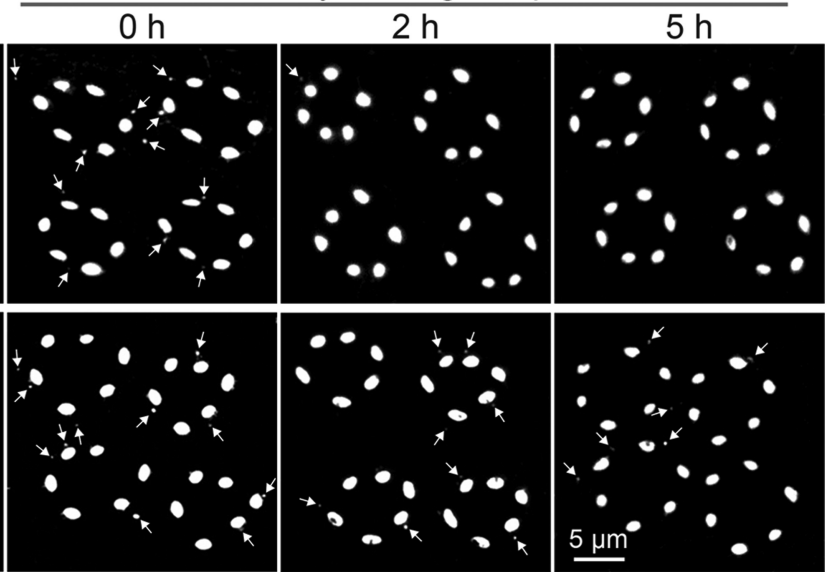

C

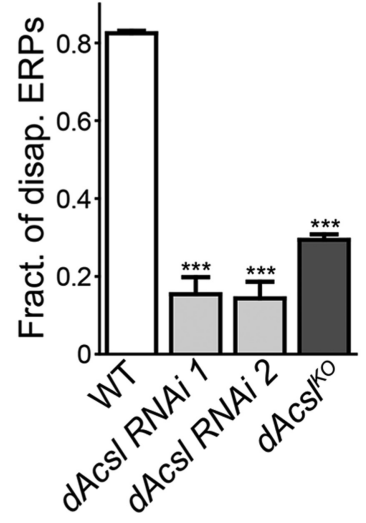

that dAcsl inhibits endocytosis of BMP receptors. However, we found no defect in synaptic vesicle (SV) endocytosis as detected by FM1-43 uptake (data not shown). Additionally, dAcsl boutons lack the typical ultrastructural features of endocytic mutants, including a low density of SVs, enlarged SVs, and an accumulation of endocytic intermediates (Verstreken et al., 2009; Shi et al., 2013; Zhao et al., 2013) (Fig. 7). Thus, dAcsl does not regulate SV endocytosis, although the possibility of a defect in receptormediated endocytosis at $d A c s l$ NMJ synapses cannot be formally ruled out.

BMP signaling can also be attenuated by targeting active receptors to lysosomes for degradation (Sweeney and Davis, 2002; Wang et al., 2007; Kim et al., 2010). However, in contrast to mutants with defects in lysosomal degradation, which show enlarged early or late endosomal compartments (Dermaut et al., 2005; Kim et al., 2010), the distribution of endosomal markers was normal at $d A c s l \mathrm{NMJ}$ synapses (Figs. 4 and 7). Thus, endosomal trafficking from early endosomes to lysosomes appears unaffected in $d A c s l$ mutant NMJs. Furthermore, in contrast to the studies in cultured human cells, where knockdown of ACSL4 results in delayed degradation of EGFR (Zhang et al., 2011), the Tkv protein level at $d A c s l$ synapses was comparable with the control (Fig. 3 ), suggesting that dAcsl may not affect Tkv degradation.

Instead, multiple lines of evidence support a model in which dAcsl attenuates BMP signaling by promoting Rab11dependent recycling and inactivation of BMP receptors (Fig. 9). First, the level of activated but not the total Tkv receptors was increased in $d A c s l$ NMJs (Fig. 3). In addition, the colocalization of Tkv receptors with the recycling endosome marker Rab11 was reduced, whereas that with early endosomal markers Rab5 and Avl/ Syx7 was increased in $d A c s l$ mutants (Fig. 5), suggesting that the trafficking of BMP receptors from early endosomes to recycling endosomes is impaired. Second, the localization of Rab11 at synaptic boutons dominant-negative form of Rab11 induced by $h s$-Gal4 at adult stage, compared with $82.5 \%$ of ERPs that disappeared after $2 \mathrm{~h}$ recovery in wild-type $(p<0.001)$. Together, these results support that dAcsl not only facilitates the recycling of BMP receptors at NMJs but also promotes the recycling of rhodopsin in the eye to maintain photoreceptor sensitivity.

\section{Discussion}

Endocytosis of BMP receptors is considered to be an efficient mechanism for limiting the amount of surface receptors (Dickman et al., 2006; O'Connor-Giles et al., 2008). Thus, a possible explanation for the elevated BMP signaling in $d A c s l$ mutants is and the membrane-cytosol cycle of Rab11 in the nervous system were altered in $d A c s l$ mutants (Figs. 4 and 7). We speculate that the expanded staining pattern of Rab11 at NMJs may be caused by the increased level of cytosolic Rab11. Consistent with this possibility, a mutated Rab5A, which lacks the membrane anchor, completely loses endosomal localization and instead shows a cytosolic and nuclear distribution in Cos-7 cells (Blümer et al., 2013), and dissociation of Rab11 from membrane redistributes Rab11 to the cytoplasm of CHO cells (Takahashi et al., 2007). The membrane association of Rab proteins is mediated by multiple interacting proteins, such as Rab escort proteins and Rab GDP- 
dissociation inhibitors, and by posttranslational covalent attachment of prenyl groups to Rabs (Seabra and Wasmeier, 2004). Prenylation of Rabs leads to a mobility change in PAGE (Detter et al., 2000). However, the mobility of Rab11 in polyacrylamide gels was normal (Figs. 4 and 7), suggesting that the prenylation of Rab11 is normal in dAcsl mutants. Thus, it is possible that a regulatory step involved in protein-protein interactions, protein-lipid interactions, or conformational changes of Rab11 might be altered in $d A c s l$ mutants. Third, $d A c s l$ synergistically interacts with rab11 to restrict synaptic overgrowth by attenuating BMP signaling (Fig. 6). Finally, dAcsl also promotes recycling of the photoreceptor rhodopsin to the rhabdomere in the eye (Fig. 8). Together, these data indicate that dAcsl regulates Rab11-dependent recycling and inactivation of the BMP receptor $\mathrm{Tkv}$ in different neuronal cell types.

How does dAcsl affect the Rab11-mediated endocytic recycling of membrane receptors? ACSL4 converts long-chain fatty acids to acyl-CoA esters. The synthetase activity of diseasecausing mutations in ACSL4 is significantly decreased (Meloni et al., 2002; Longo et al., 2003), suggesting that reduction of the enzyme activity of ACSL4 is responsible for the pathogenesis of ACSL4-related mental retardation. Indeed, expression of these mutated ACSL4 failed to rescue the mistargeting of the retinal axons in the brains (Zhang et al., 2009), the NMJ growth defects, and the altered Rab11 localization in $d A c s l$ mutants (data not shown). These data suggest that the acyl-CoA synthetase activity may be responsible for the endocytic recycling defects. ACSL4 has a strong preference for polyunsaturated fatty acids, such as arachidonic acid (C20:4) and eicosatetraenoic acid (C20:5) (Kang et al., 1997; Cao et al., 1998). Reduced incorporation of C20:4 and C20:5 into phospholipids may change membrane properties, such as bilayer fluidity and membrane protein targeting (Escribá et al., 2008), and may underlie the decreased membrane association of Rab11. The altered membrane association of Rab11, but not Rab5, suggests that dAcsl might regulate lipid composition of specific membrane compartments. Indeed, lipid composition specifically affects membrane association of different Rabs (e.g., cholesterol specifically affects membrane-cytosol cycle of Rab7 but not Rab5) (Lebrand et al., 2002). In addition to the enrichment of dAcsl in ER, the major site for lipid biosynthesis (Zhang et al., 2009; O'Sullivan et al., 2012), we also observed colocalization between dAcsl and Tkv-GFP, as well as the endosomal markers Rab5 and Rab11 at NMJs (Fig. 5), indicating that dAcsl might act locally in regulating endocytic trafficking. The exact mechanisms by which dAcsl regulates Rab11-mediated endocytic recycling, however, remain to be determined.

Despite the obvious overgrowth of NMJs in the anterior segments, the NMJs in the posterior segments were dystrophic (Fig. 1) (Liu et al., 2011). This distinct bidirectional NMJ phenotype in $d A c s l$ mutants, to our knowledge, has not been previously reported. The overgrown NMJs in the anterior segments can be readily explained by increased BMP signaling. But how are the dystrophic NMJs in the posterior segments of
dAcsl mutants brought about? We observed that the levels of pMad and activated Tkv receptors were increased, and the staining pattern of Rab11 was altered in the posterior NMJs of dAcsl mutants. Thus, the Rab11-dependent trafficking of the $\mathrm{BMP}$ receptor Tkv in the posterior NMJs of $d A c s l$ mutants was also defective. However, as a result of the axonal transport defects (Liu et al., 2011), the increased levels of activated Tkv and pMad at the posterior NMJs and other retrograde cargoes could not be efficiently transported back to the cell bodies of motoneurons, or the anterograde transport of lipids, proteins, and organelles to the synaptic terminals is impaired, or both, resulting in malnourished posterior NMJs in AAcsl mutants. Consistent with this scenario, it has been previously shown that the longer the axons, the more susceptible the NMJ growth to axonal transport defects (Hurd and Saxton, 1996). Thus, the upregulated BMP signaling at NMJs across different segments and the increased susceptibility of posterior NMJs to axonal transport defects may cause the unique overgrowth-to-undergrowth phenotype of NMJs in dAcsl mutants.

In the larval brains of $d A c s l$ mutants, the production of decapentaplegic (Dpp, a BMP-like molecule) is diminished, although $\mathrm{dAcsl}$ is not intrinsically required for Dpp expression (Zhang et al., 2009). Consistent with the Dpp reduction, the number of glial cells and neurons is decreased, and the retinal axons are mistargeted in the visual cortex (Zhang et al., 2009). In contrast to the reduced production of Dpp in the brains, we observed elevated BMP signaling at the peripheral NMJ synapses of $d A c s l$ mutants (Fig. 3). Thus, dAcsl regulates BMP signaling differentially in different cellular texts. The underlying mechanisms for the differential regulation of BMP pathway by dAcsl await further investigations.

In conclusion, this study provides experimental evidence implicating dAcsl in synaptic development and endocytic trafficking of BMP receptors via recycling endosomes and offers novel insight into the pathogenesis of mental retardation caused by mutations in ACSL4. Given that several genes involved in the synthesis and turnover of long-chain fatty acids affect brain development and cognitive function (Najmabadi et al., 2011), our findings may also help understand the neurodevelopmental defects caused by mutations in other genes involved in lipid metabolism pathways. 


\section{References}

Bayat V, Jaiswal M, Bellen HJ (2011) The BMP signaling pathway at the Drosophila neuromuscular junction and its links to neurodegenerative diseases. Curr Opin Neurobiol 21:182-188. CrossRef Medline

Blümer J, Rey J, Dehmelt L, Mazel T, Wu YW, Bastiaens P, Goody RS, Itzen A (2013) RabGEFs are a major determinant for specific Rab membrane targeting. J Cell Biol 200:287-300. CrossRef Medline

Cao J, Li Y, Xia W, Reddig K, Hu W, Xie W, Li HS, Han J (2011) A Drosophila metallophosphoesterase mediates deglycosylation of rhodopsin. EMBO J 30:3701-3713. CrossRef Medline

Cao Y, Traer E, Zimmerman GA, McIntyre TM, Prescott SM (1998) Cloning, expression, and chromosomal localization of human long-chain fatty acid-CoA ligase 4 (FACL4). Genomics 49:327-330. CrossRef Medline

Cao Y, Murphy KJ, McIntyre TM, Zimmerman GA, Prescott SM (2000) Expression of fatty acid-CoA ligase 4 during development and in brain. FEBS Lett 467:263-267. CrossRef Medline

Collins CA, DiAntonio A (2007) Synaptic development: insights from Drosophila. Curr Opin Neurobiol 17:35-42. CrossRef Medline

Coyle IP, Koh YH, Lee WC, Slind J, Fergestad T, Littleton JT, Ganetzky B (2004) Nervous wreck, an SH3 adaptor protein that interacts with Wsp, regulates synaptic growth in Drosophila. Neuron 41:521-534. CrossRef Medline

Dermaut B, Norga KK, Kania A, Verstreken P, Pan H, Zhou Y, Callaerts P, Bellen HJ (2005) Aberrant lysosomal carbohydrate storage accompanies endocytic defects and neurodegeneration in Drosophila benchwarmer. J Cell Biol 170:127-139. CrossRef Medline

Detter JC, Zhang Q, Mules EH, Novak EK, Mishra VS, Li W, McMurtrie EB, Tchernev VT, Wallace MR, Seabra MC, Swank RT, Kingsmore SF (2000) Rab geranylgeranyl transferase alpha mutation in the gunmetal mouse reduces Rab prenylation and platelet synthesis. Proc Natl Acad Sci U S A 97:4144-4149. CrossRef Medline

Dickman DK, Lu Z, Meinertzhagen IA, Schwarz TL (2006) Altered synaptic development and active zone spacing in endocytosis mutants. Curr Biol 16:591-598. CrossRef Medline

Dudu V, Bittig T, Entchev E, Kicheva A, Jülicher F, González-Gaitán M (2006) Postsynaptic mad signaling at the Drosophila neuromuscular junction. Curr Biol 16:625-635. CrossRef Medline

Escribá PV, González-Ros JM, Goñi FM, Kinnunen PK, Vigh L, SánchezMagraner L, Fernández AM, Busquets X, Horváth I, Barceló-Coblijn G (2008) Membranes: a meeting point for lipids, proteins and therapies. J Cell Mol Med 12:829-875. CrossRef Medline

Fuentes-Medel Y, Budnik V (2010) Ménage à Trio during BMP-mediated retrograde signaling at the NMJ. Neuron 66:473-475. CrossRef Medline

Grant BD, Donaldson JG (2009) Pathways and mechanisms of endocytic recycling. Nat Rev Mol Cell Biol 10:597-608. CrossRef Medline

Han J, Reddig K, Li HS (2007) Prolonged G(q) activity triggers fly rhodopsin endocytosis and degradation, and reduces photoreceptor sensitivity. EMBO J 26:4966-4973. CrossRef Medline

Hurd DD, Saxton WM (1996) Kinesin mutations cause motor neuron disease phenotypes by disrupting fast axonal transport in Drosophila. Genetics 144:1075-1085. Medline

Kang MJ, Fujino T, Sasano H, Minekura H, Yabuki N, Nagura H, Iijima H, Yamamoto TT (1997) A novel arachidonate-preferring acyl-CoA synthetase is present in steroidogenic cells of the rat adrenal, ovary, and testis. Proc Natl Acad Sci U S A 94:2880-2884. CrossRef Medline

Khodosh R, Augsburger A, Schwarz TL, Garrity PA (2006) Bchs, a BEACH domain protein, antagonizes Rab11 in synapse morphogenesis and other developmental events. Development 133:4655-4665. CrossRef Medline

Kim S, Wairkar YP, Daniels RW, DiAntonio A (2010) The novel endosomal membrane protein Ema interacts with the class C Vps-HOPS complex to promote endosomal maturation. J Cell Biol 188:717-734. CrossRef Medline

Koles K, Nunnari J, Korkut C, Barria R, Brewer C, Li Y, Leszyk J, Zhang B, Budnik V (2012) Mechanism of evenness interrupted (Evi)-exosome release at synaptic boutons. J Biol Chem 287:16820-16834. CrossRef Medline

Krämer H, Phistry M (1996) Mutations in the Drosophila hook gene inhibit endocytosis of the boss transmembrane ligand into multivesicular bodies. J Cell Biol 133:1205-1215. CrossRef Medline

Lebrand C, Corti M, Goodson H, Cosson P, Cavalli V, Mayran N, Fauré J, Gruenberg J (2002) Late endosome motility depends on lipids via the small GTPase Rab7. EMBO J 21:1289-1300. CrossRef Medline
Li BX, Satoh AK, Ready DF (2007) Myosin V, Rab11, and dRip11 direct apical secretion and cellular morphogenesis in developing Drosophila photoreceptors. J Cell Biol 177:659-669. CrossRef Medline

Li LO, Klett EL, Coleman RA (2010) Acyl-CoA synthesis, lipid metabolism and lipotoxicity. Biochim Biophys Acta 1801:246-251. CrossRef Medline

Liu Z, Huang Y, Zhang Y, Chen D, Zhang YQ (2011) Drosophila Acyl-CoA synthetase long-chain family member 4 regulates axonal transport of synaptic vesicles and is required for synaptic development and transmission. J Neurosci 31:2052-2063. CrossRef Medline

Longo I, Frints SG, Fryns JP, Meloni I, Pescucci C, Ariani F, Borghgraef M, Raynaud M, Marynen P, Schwartz C, Renieri A, Froyen G (2003) A third MRX family (MRX68) is the result of mutation in the long chain fatty acid-CoA ligase 4 (FACL4) gene: proposal of a rapid enzymatic assay for screening mentally retarded patients. J Med Genet 40:11-17. CrossRef Medline

Meloni I, Muscettola M, Raynaud M, Longo I, Bruttini M, Moizard MP, Gomot M, Chelly J, des Portes V, Fryns JP, Ropers HH, Magi B, Bellan C, Volpi N, Yntema HG, Lewis SE, Schaffer JE, Renieri A (2002) FACL4, encoding fatty acid-CoA ligase 4 , is mutated in nonspecific X-linked mental retardation. Nat Genet 30:436-440. CrossRef Medline

Meloni I, Parri V, De Filippis R, Ariani F, Artuso R, Bruttini M, Katzaki E, Longo I, Mari F, Bellan C, Dotti CG, Renieri A (2009) The XLMR gene ACSL4 plays a role in dendritic spine architecture. Neuroscience 159:657669. CrossRef Medline

Michel M, Raabe I, Kupinski AP, Pérez-Palencia R, Bökel C (2011) Local BMP receptor activation at adherens junctions in the Drosophila germline stem cell niche. Nat Commun 2:415. CrossRef Medline

Nahm M, Lee MJ, Parkinson W, Lee M, Kim H, Kim YJ, Kim S, Cho YS, Min BM, Bae YC, Broadie K, Lee S (2013) Spartin regulates synaptic growth and neuronal survival by inhibiting BMP-mediated microtubule stabilization. Neuron 77:680-695. CrossRef Medline

Najmabadi H, Hu H, Garshasbi M, Zemojtel T, Abedini SS, Chen W, Hosseini M, Behjati F, Haas S, Jamali P, Zecha A, Mohseni M, Püttmann L, Vahid LN, Jensen C, Moheb LA, Bienek M, Larti F, Mueller I, Weissmann R, et al. (2011) Deep sequencing reveals 50 novel genes for recessive cognitive disorders. Nature 478:57-63. CrossRef Medline

Ni JQ, Liu LP, Binari R, Hardy R, Shim HS, Cavallaro A, Booker M, Pfeiffer BD, Markstein M, Wang H, Villalta C, Laverty TR, Perkins LA, Perrimon N (2009) A Drosophila resource of transgenic RNAi lines for neurogenetics. Genetics 182:1089-1100. CrossRef Medline

O'Connor-Giles KM, Ho LL, Ganetzky B (2008) Nervous wreck interacts with thickveins and the endocytic machinery to attenuate retrograde BMP signaling during synaptic growth. Neuron 58:507-518. CrossRef Medline

O'Sullivan NC, Jahn TR, Reid E, O'Kane CJ (2012) Reticulon-like-1, the Drosophila ortholog of the Hereditary Spastic Paraplegia gene reticulon 2, is required for organization of endoplasmic reticulum and of distal motor axons. Hum Mol Genet 21:3356-3365. CrossRef Medline

Otani T, Oshima K, Onishi S, Takeda M, Shinmyozu K, Yonemura S, Hayashi S (2011) IKKepsilon regulates cell elongation through recycling endosome shuttling. Dev Cell 20:219-232. CrossRef Medline

Persson U, Izumi H, Souchelnytskyi S, Itoh S, Grimsby S, Engström U, Heldin CH, Funa K, ten Dijke P (1998) The L45 loop in type I receptors for TGF-beta family members is a critical determinant in specifying Smad isoform activation. FEBS Lett 434:83-87. CrossRef Medline

Pulipparacharuvil S, Akbar MA, Ray S, Sevrioukov EA, Haberman AS, Rohrer J, Krämer H (2005) Drosophila Vps16A is required for trafficking to lysosomes and biogenesis of pigment granules. J Cell Sci 118:3663-3673. CrossRef Medline

Rodal AA, Blunk AD, Akbergenova Y, Jorquera RA, Buhl LK, Littleton JT (2011) A presynaptic endosomal trafficking pathway controls synaptic growth signaling. J Cell Biol 193:201-217. CrossRef Medline

Satoh AK, Ready DF (2005) Arrestin1 mediates light-dependent rhodopsin endocytosis and cell survival. Curr Biol 15:1722-1733. CrossRef Medline

Seabra MC, Wasmeier C (2004) Controlling the location and activation of Rab GTPases. Curr Opin Cell Biol 16:451-457. CrossRef Medline

Shi W, Chen Y, Gan G, Wang D, Ren J, Wang Q, Xu Z, Xie W, Zhang YQ (2013) Brain tumor regulates neuromuscular synapse growth and endocytosis in Drosophila by suppressing mad expression. J Neurosci 33: 12352-12363. CrossRef Medline

Sorkin A, von Zastrow M (2009) Endocytosis and signalling: intertwining 
molecular networks. Nat Rev Mol Cell Biol 10:609-622. CrossRef Medline

Stenmark H (2009) Rab GTPases as coordinators of vesicle traffic. Nat Rev Mol Cell Biol 10:513-525. CrossRef Medline

Stowers RS, Schwarz TL (1999) A genetic method for generating Drosophila eyes composed exclusively of mitotic clones of a single genotype. Genetics 152:1631-1639. Medline

Sweeney ST, Davis GW (2002) Unrestricted synaptic growth in spinster: a late endosomal protein implicated in TGF-beta-mediated synaptic growth regulation. Neuron 36:403-416. CrossRef Medline

Takahashi M, Murate M, Fukuda M, Sato SB, Ohta A, Kobayashi T (2007) Cholesterol controls lipid endocytosis through Rab11. Mol Biol Cell 18: 2667-2677. CrossRef Medline

Tanaka T, Nakamura A (2008) The endocytic pathway acts downstream of Oskar in Drosophila germ plasm assembly. Development 135:1107-1117. CrossRef Medline

Verstreken P, Ohyama T, Haueter C, Habets RL, Lin YQ, Swan LE, Ly CV, Venken KJ, De Camilli P, Bellen HJ (2009) Tweek, an evolutionarily conserved protein, is required for synaptic vesicle recycling. Neuron 63: 203-215. CrossRef Medline

Wang X, Shaw WR, Tsang HT, Reid E, O'Kane CJ (2007) Drosophila spichthyin inhibits BMP signaling and regulates synaptic growth and axonal microtubules. Nat Neurosci 10:177-185. CrossRef Medline

Wucherpfennig T, Wilsch-Bräuninger M, González-Gaitán M (2003) Role of Drosophila Rab5 during endosomal trafficking at the synapse and evoked neurotransmitter release. J Cell Biol 161:609-624. CrossRef Medline

Zhang C, Li A, Zhang X, Xiao H (2011) A novel TIP30 protein complex regulates EGF receptor signaling and endocytic degradation. J Biol Chem 286:9373-9381. CrossRef Medline

Zhang Y, Chen D, Wang Z (2009) Analyses of mental dysfunction-related ACS14 in Drosophila reveal its requirement for Dpp/BMP production and visual wiring in the brain. Hum Mol Genet 18:3894-3905. CrossRef Medline

Zhao L, Wang D, Wang Q, Rodal AA, Zhang YQ (2013) Drosophila cyfip regulates synaptic development and endocytosis by suppressing filamentous actin assembly. Plos Genet 9:e1003450. CrossRef Medline 